\title{
HÍBRIDOS NUEVOS EN EL GÉNERO TEUCRIUM L. (LAMIACEAE)
}

\author{
Antonio DE LA TORRE y Francisco ALCARAZ
}

\begin{abstract}
RESUMEN. Se describen cuatro nuevas nothoespecies del género Teucrium L. (Sect. Polium) para el sureste de España: $T$. x rigualii (T. libanitis $x T$. carolipaui), $T$. $x$ riosii ( $T$. capitatum subsp. gracillimum $x$ T.carolipaui), T.x riverae (T. capitatum subsp. gracillimum $x T$.dunense), yT. x robledoi (T. capitatum subsp. gracillimum xT. homotrichum). Asimismo se aportan datos sobre la posición bioclimática, biogeográfica y fitosociológica de cada uno de ellos.
\end{abstract}

Palabras clave. Teucrium, nothoespecies, híbridos, flora, España.

ABSTRACT. Four new nothospecies are described within the genus Teucrium L. (Sect. Polium), from southeastern Spain: $T$. x rigualii (T. libanitis $x$ T. carolipaui), $T$. x riosii (T. capitatum subsp. gracillimum $x$ T.carolipaui), T.x riverae (T. capitatum subsp. gracillimum $x T$.dunense), and T. $x$ robledoi ( $T$. capitatum subsp. gracillimum $x T$. homotrichum). Data on the bioclimatology, biogeography and phytosociology for all these hybrids is also given.

Key word. Teucrium, nothospecies, hybrids, flora, Spain.

\section{INTRODUCCIÓN}

Bien conocida es la facilidad de las especies del género Teucrium para dar híbridos, como se manifiesta en la abundancia de nototáxones descritos (Font Quer, 1926, 1946; Sennen, 1936; Alcaraz, Sánchez-Gómez y Carrión, 1988; Pajarón y Molina, 1989; Crespo y Mateo, 1991). Parecen ser más dados a ello los representantes de la sección Polium en la que el área parcialmente simpátrica de sus táxones facilita el cruzamiento (Puech, 1984).

Concretamente, en la provincia de Alicante y, posteriormente en otras localidades del sureste ibérico, se han encontrado poblaciones hibridógenas habitando entre sus parentales, en las que se aprecian características intermedias delatadoras de la hibridación. Teucrium capitatum L. subsp. gracillimum se ha mostrado, una vez más como muy proclive a la hibridación con especies próximas de su misma sección: $T$. homotrichum, T. carolipaui, T. dunense, T. murcicum (Alcaraz et al., 1991), T. angustissimum (Crespo y Mateo, 1991).

En el presente trabajo se describen cuatro notoespecies pertenecientes al género Teucrium a partir de material recolectado en la provincia de Alicante. El material tipo ha quedado depositado en el Herbario de la Universidad de Murcia (MUB), material isotípico ha sido depositado en el Herbario de la Universidad de Alicante $(\mathrm{ABH})$. En los resultados se indica el número de herbario de todos los pliegos. 


\section{MATERIAL Y MÉTODOS}

Para la nomenclatura de los táxones híbridos propuestos se siguen las normas del Código de Nomenclatura Botánica (Greuter et al., 1988). Las autorías de los táxones que aparecen están en concordancia con las propuestas de Navarro y Rosúa (1990).

Los aspectos corológicos y bioclimáticos se presentan de acuerdo a las directrices de Alcaraz et al. (1991) y De la Torre (1991).

\section{RESULTADOS Y DISCUSIÓN}

Teucrium x robledoi De la Torre et Alcaraz, nothosp. nov. [Teucrium homotrichum (Font Quer) Rivas-Martínez $x T$. capitatum L. subsp. gracillimum (Rouy) Valdés-Bermejo].

Holotypus: Hs, Alicante: Sierra de la Solana (Beneixama), 30SXH9290, ad $800 \mathrm{~m}$ alt., inter parentes in collibus aridis calcareis; 18-VI-1987, A. de la Torre legit (MUB 21655).

Isotypus: ibidem (ABH 1000).

A Teucrio homotricho, inflorescentia composita, floribus in glomerulis longe pedunculatis $(1 \mathrm{~cm})$, corolla rosea differt. A Teucrio capitato subsp. gracillimo glomerulis floralibus majoribus $(1-1,5 \mathrm{~cm})$, foliis latioribus $(3-5 \mathrm{~mm})$, pilis coralliformibus differt.

Species hybrida botanico amico Antonio Robledo dicata est.

Icon: Fig. 1.

Difiere de T. homotrichum por su inflorescencia ramificada, pedúnculos de las cabezuelas de hasta $1 \mathrm{~cm}$ y flores rosadas. Difiere de $T$. capitatum subsp. gracillimum por presentar las cabezuelas mayores, de al menos $1 \mathrm{~cm}$ de diámetro, pelos coraliformes y hojas más anchas, de 3 a $5 \mathrm{~mm}$ de anchura.

Muy raro, en matorrales que colonizan las cunetas de pi:las forestales, en las que abunda Teucrium capitatum subsp. gracillimum. Dada el árcia de los dos táxones, el híbrido debe tener una distribución suroriental ibérica, en las provincias de Alicante y Valencia, donde todavía no ha sido encontrado. Se le conoce de los horizontes medio y superior del piso Mesomediterráneo, con ombroclima semiárido a subhúmedo. Ecológicamente tiene sus óptimo entre los matorrales calcícolas setabenses (TeucrioThymenion piperellae Stübing, Peris y Costa 1989) y los herbazales subnitrófilos viarios del orden Helichryso-Santolinetalia Peinado y Martínez-Parras 1984.

Teucrium $\mathrm{x}$ riosii De la Torre et Alcaraz nothosp. nov. [Teucrium carolipaui C. Vicioso ex Pau subsp. carolipaui x Teucrium capitatum L. subsp. gracillimum (Rouy) Valdés-Bermejo]

Holotypus: Hs, Alicante: Solana del Fraile (Biar), 30SXH9375, ad 680 m alt., inter parentes in collibus aridis calcareis; 3-VII-1987, A. de la Torre legit (MUB 21662).

Isotypus: ibidem (ABH 1001).

A Teucrio carolipaui subsp. carolipaui bracteis brevioribus, floribus longitudinis aequantibus in glomerulis minoribus $(<1 \mathrm{~cm})$ dispositis differt. A Teucrio 
capitato subsp. gracillimofoliis subglabris viridis, calycibus glandulosis nec tomentosis differt.

Species hybrida botanico amico Segundo Ríos dicata est.

Icon: Fig. 2.

Difiere de T. capitatum subsp. gracillimum por sus hojas verdes, subglabras y cálices punteado-glandulosos, no tomentosos. Difiere de T. carolipaui por las brácteas subiguales al cáliz y cabezuelas menores de $1 \mathrm{~cm}$.

Planta abundante en los tomillares calcícolas termófilos de la mitad sur de la provincia de Alicante y áreas limítrofes de Murcia (Thymo-Siderition leucanthae O. Bolòs 1957). Bioclimáticamente se sitúa entre el horizonte superior del piso Termomediterráneo y el inferior del piso Mesomediterráneo, siempre en áreas de ombroclima semiárido.

Sennen (1936) describió en su «Note $1^{\mathfrak{a}}{ }$ al número 7189 de la serie de 1929 un híbrido entre Teucrium fontqueri Sennen [Teucrium carolipaui subsp. fontqueri (Sennen) Rivas-Martínez] y Teucrium angustifolium Duf., al que llamó Teucrium salgadoi Sennen y que había recolectado entre Lorca y Carraclaca (Murcia). Según hemos comprobado, en esta localidad murciana conviven T. carolipaui subsp. fontqueri, $T$. murcicum y $T$. capitatum subsp. gracillimum, por lo que resulta difícil suponer la identidad de T. $x$ salgadoi sin estudiar el material tipo, incluso a la vista de la escueta y poco clarificadora diagnosis ofrecida por Sennen. A fin de corroborar la identidad de tal híbrido, que podría incluso obligar a describir Teucrium $x$ riosii como una nothosubespecie del citado Teucrium $x$ salgadoi, se buscaron los pliegos de tal recolección en el Herbario del Instituto Botánico de Barcelona (BC) y en el Herbario de Sennen del Colegio La Salle Bonanova de Barcelona, con resultados negativos en ambos casos. Por tanto no se conoce material del citado híbrido de Sennen, ante lo cual y, siempe sujetos a la posibilidad de que en el futuro pudiera aparecer algún pliego que respaldara el nombre senneniano, se ha optado por describir Teucrium x riosii como una nueva nothoespecie.

Teucrium x riverae De la Torre et Alcaraz nothosp. nov.

[Teucrium dunense Sennen $x$ Teucrium capitatum L. subsp. gracillimum (Rouy) ValdésBermejo]

Holotypus: Hs, Alicante: Solana del Fraile (Biar), 30SXH9475, ad 660 m alt., inter parentes in collibus aridis sabulosis; 3-VII-1987, A. de la Torre legit (MUB 21659).

Isotypus: ibidem (ABH 1002).

A Teucrio dunense glomerulis floralibus minoribus (7-9 mm), corolla rosea, calycibus non cucullatis differt. A Teucrio capitato subsp. gracillimo glomerulis floralibus majoribus (>6 mm), calycibus $4-5 \mathrm{~mm}$ long. differt.

Species hybrida botanico amico Diego Rivera dicata est.

Icon: Fig. 3.

Difiere de $T$. dunense por presentar cabezuelas menores, de hasta $9 \mathrm{~mm}$ de diámetro, flores rosadas y cáliz no cuculado. Difiere de T. capitatum subsp. gracillimum por presentar cabezuelas mayores de $6 \mathrm{~mm}$ y cáliz de más de $4 \mathrm{~mm}$ de longitud. 
Relativamente abundante en las áreas donde existen depósitos cuaternarios de arenas calcáreas, frecuentes en el interior de la provincia de Alicante, donde forma parte de matorrales calcícolas en contacto con matorrales sabulícolas. Ha sido observado en los horizontes inferior y medio del piso Mesomediterráneo, con ombroclima semiárido, en el seno de comunidades camefíticas y nanofanerofíticas de la subalianza Teucrio-Thymenion piperellae.

Teucrium x rigualii De la Torre et Alcaraz nothosp. nov. [T. libanitis Schreber $x$ T. carolipaui C. Vicioso ex Pau subsp. carolipaui].

Holotypus: Hs, Alicante: Sierra del Maigmó (Agost), 30SYH0662, ad 500 m, inter parentes in collibus aridis gypsaceis; 3-VII-1987, A. de la Torre legit (MUB 14869).

Isotypus: ibidem (ABH 1003).

A Teucrio libanitidis foliis oppositis, caulibus subglabris differt nec tomentosis. A Teucrio carolipaui subsp. carolipaui bracteis floribus longitudinis aequantibus, subtus cano-tomentellis differt.

Species hybrida magistro botanico Abelardo Rigual dicata est.

Icon: Fig. 4.

Difiere de Teucrium libanitis por presentar hojas opuestas, tallos no densamente lanoso-tomentosos. Difiere de Teucrium carolipaui por las brácteas subiguales a las flores, blanco-tomentosas por el envés.

Caméfito sufruticoso abundante en los diapiros del Keuper de los territorios alicantinos meridionales, donde forma parte de los tomillares gipsícolas (ThymoTeucrienion libanitidis [[Rivas Goday] Rivas Goday y Rivas-Martínez 1969] Alcaraz et al. 1991). Ha sido observado en las cercanías de Agost y de Elche, dónde se detectó por vez primera, en cualquier caso, siempre entre los horizontes superior, del piso Termomediterráneo e inferior del Mesomediterráneo, en condiciones ómbricas semiáridas.

AGRADECIMIENTOS. Deseamos expresar nuestro agradecimiento al Dr. M. B. Crespo por su ayuda en las descripciones latinas, así como por sus observaciones sobre algunos aspectos de la ecología de los híbridos descritos.

Agradecemos a Angel Romo (Institut Botánic de Barcelona) sus orientaciones en la búsqueda de los pliegos de Sennen correspondientes a posibles híbridos.

Asimismo queremos reconocer nuestro más sincero agradecimiento al Hermano Lamberto Munt (Colegio La Salle Bonanova de Barcelona) por su colaboración en la búsqueda del citado material de Sennen.

\section{BIBLIOGRAFÍA}

ALCARAZ, F., P. SÁNCHEZ-GÓMEZ, y J.S. CARRIÓN -1988- Teucrium x estevei Alcaraz, Sánchez-Gómez y Carrión hybr. nov. Lazaroa, 9: 25-30.

ALCARAZ, F., P. SÁNCHEZ-GÓMEZ, A. DE LA TORRE, S. M y J. ALVAREZ-ROGEL -1991-. Datos sobre la vegetación de Murcia (España). DM-PPU editores.

CRESPO, M.B. y G. MATEO -1991-. New Spanish nothotaxa in the genus Teucrium L. (Lamiaceae). Fl. Medit., 1: 195-203. 
DE LA TORRE, A. -1991-. Vegetación y suelos de la cuenca del Vinalopó (Alicante). Tesis Doctoral, inéd. Facultad de Biología. Universidad de Murcia.

FONT QUER, P. -1926-. Illustrationes florae occidentalis, I. «Mus. Ciènc. Nat.», 8 pp., 12 taul., Barcelona.

FONT QUER, P. -1946-. «Sertulum cavanillesianum enneanthum». Anales Jard. Bot. Madrid, 6(2): 487-495.

GREUTER, W., H.M. BURDET, W.G. CHALONER, V. DEMOULIN, R. GROLLE, D.L. HAWKS-WORTH, D.H. NICOLSON, P.C. SILVA, F.A. STAFLEU, E.G. VOSS \& J. MCNEILL -1988-. International Code of Botanical Nomenclature. Regnum vegetabile 118. Koeltz Scientific Books, Königstein.

PAJARÓN, S. y A. MOLINA -1989-. Teucrium x scorolepis, nuevo híbrido de la sección Scorodonia. Fontqueria, 27: 163-164.

PUECH, S. -1984- Les Teucrium (Labiées) de la section Polium du bassin méditerranéen occidental (France et péninsule ibérique). Naturalia Monspel., Fasc. Hors Sér. [1].

SENNEN, F. -1936-. Diagnoses de nouveautes parues dans les exsiccata. Plantes d'Espagne et du Maroc de 1928 a 1935. Imp. Anglada Vic.

(Aceptado para su publicación en Mayo de 1992)

Dirección de los autores. A. de la Torre: División de Biología Animal y Vegetal (Botánica), Dpto. Ciencias Ambientales y Recursos Naturales, Universidad de Alicante, 03080 Alicante. F. Alcaraz: Dpto. de Biología Vegetal (Botánica), Universidad de Murcia 30100 Murcia. 


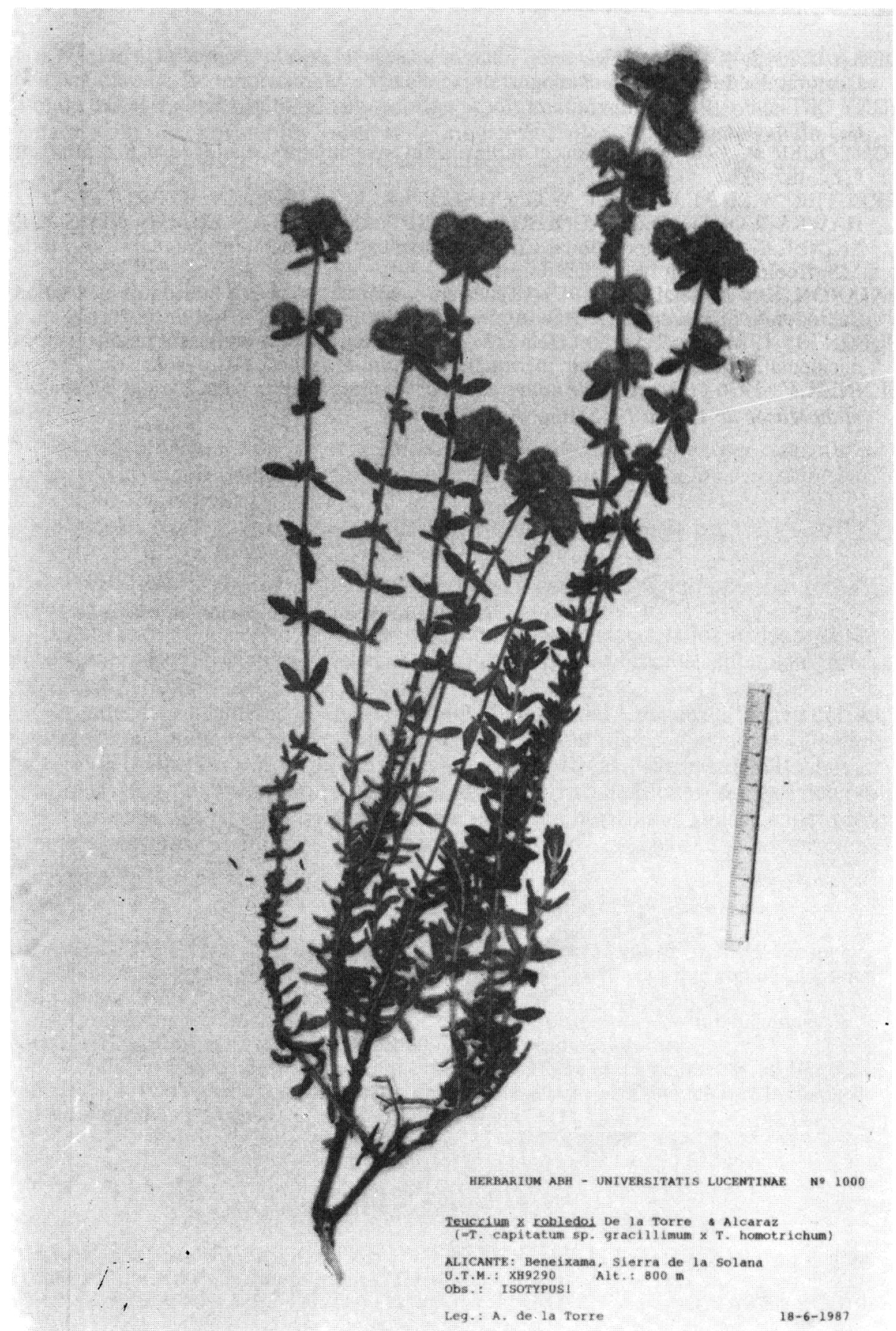

Figura 1: Isotypus de Teucrium x robledoi De la Torre et Alcaraz (ABH 1000). 


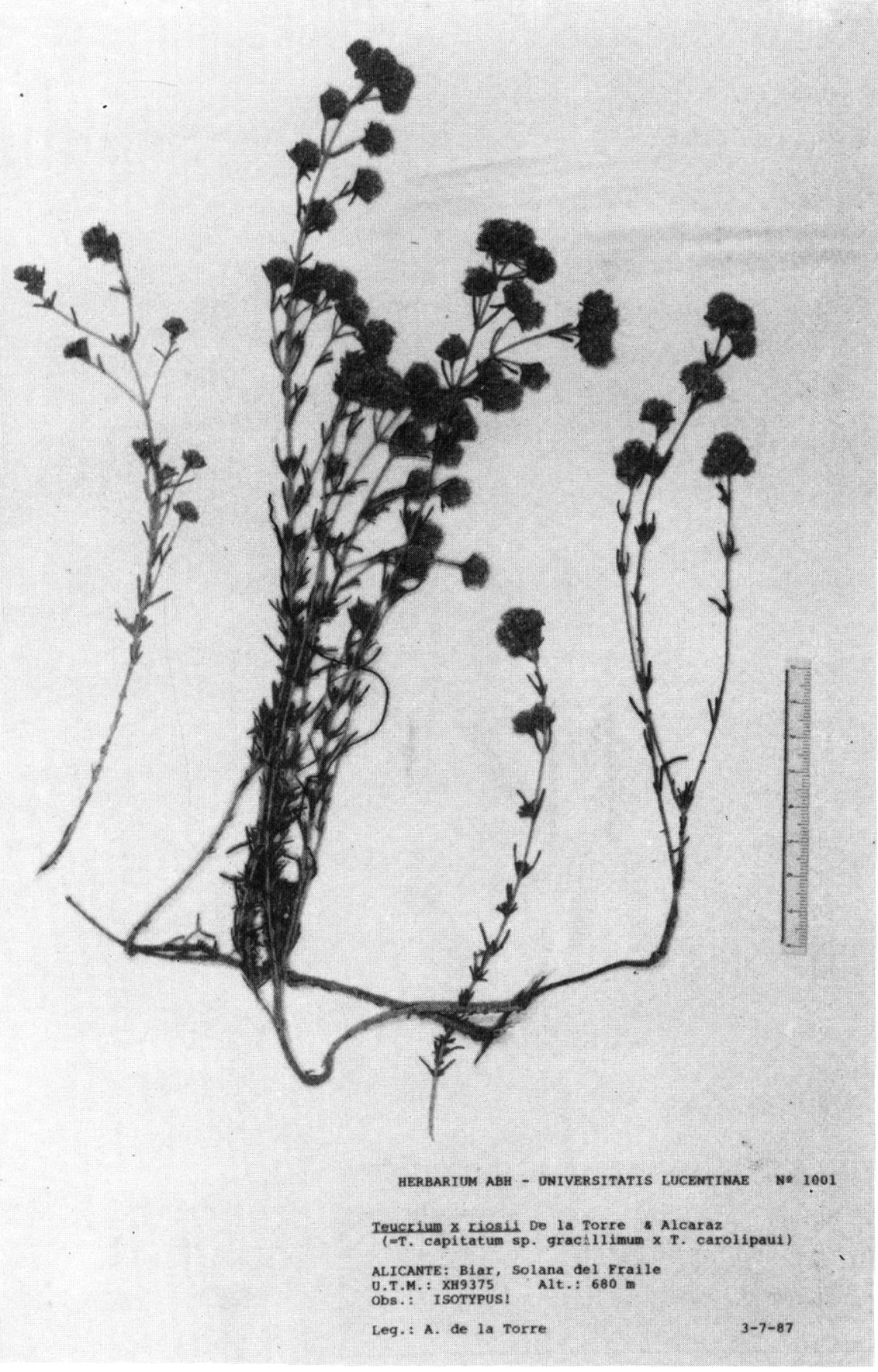

Figura 2: Isotypus de Teucrium x riosii De la Torre et Alcaraz (ABH 1001). 


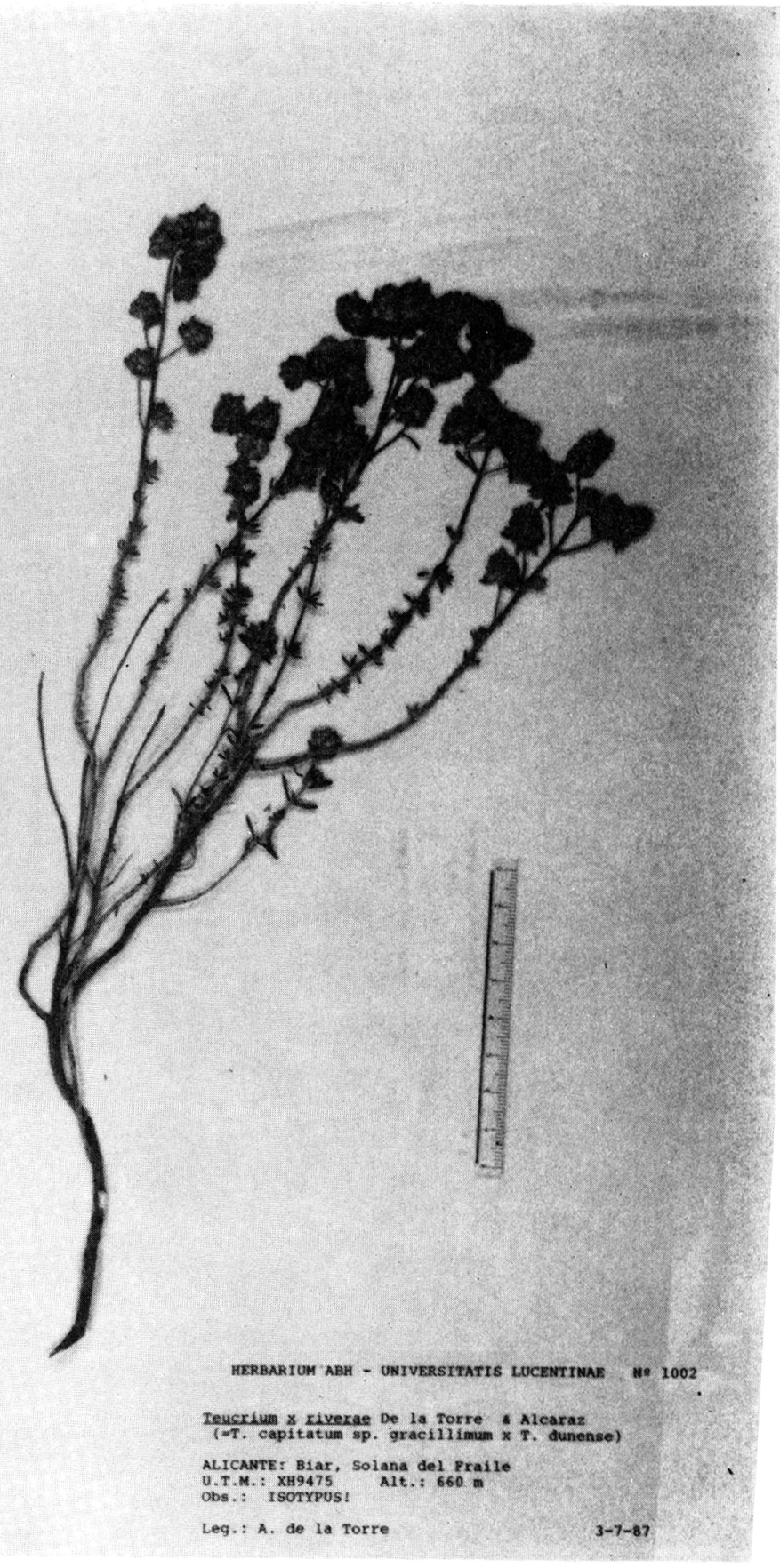

Figura 3: Isotypus de Teucrium x riverae De la Torre et Alcaraz (ABH 1002). 


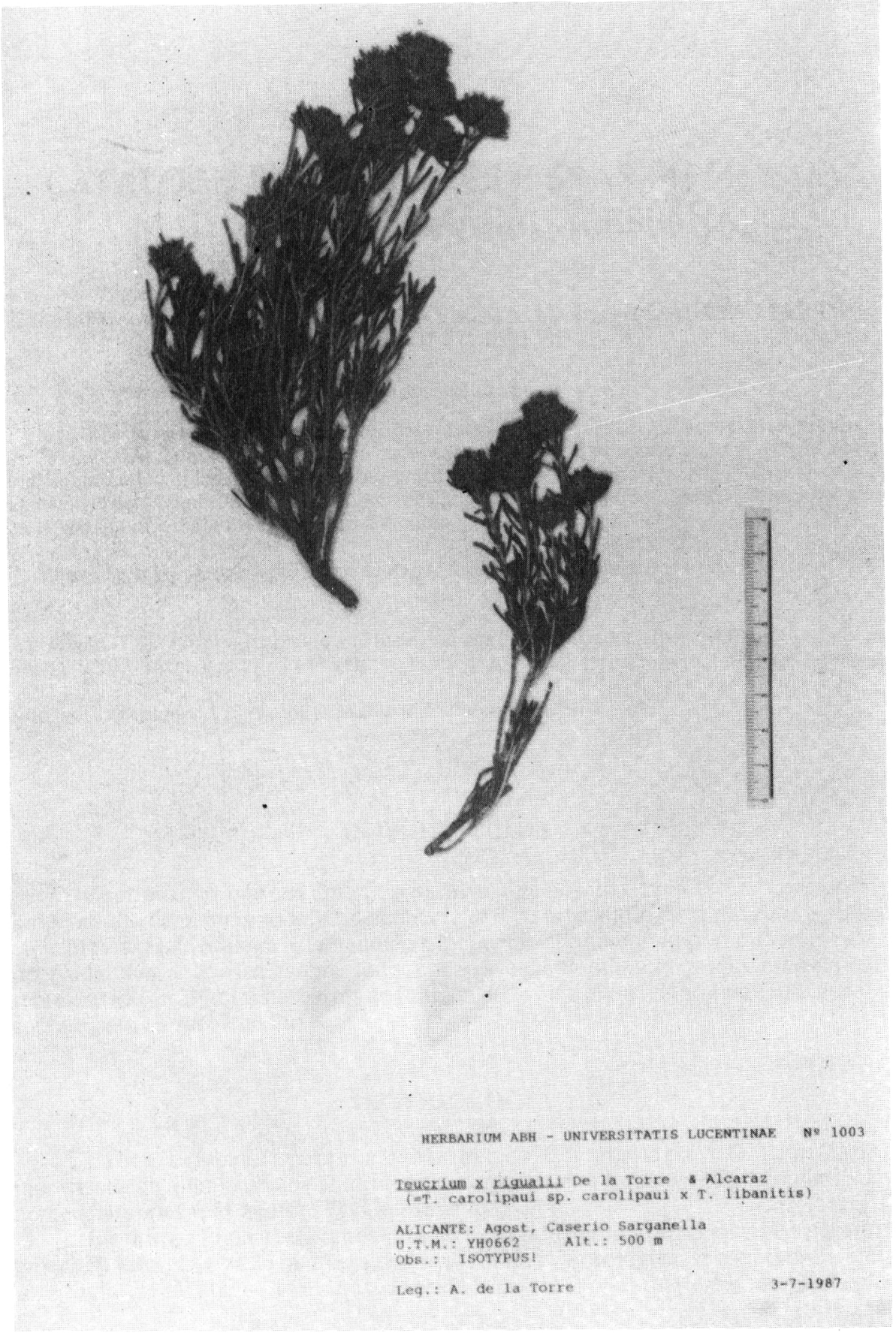

Figura 4: Isotypus de Teucrium x rigualii De la Torre et Alcaraz (ABH 1003). 